\title{
A Technique for Code Generation of USN Applications Based on Nano-Qplus*
}

\author{
Kwangyong Lee ${ }^{1}$, Woojin Lee $^{2}$, Juil Kim² ${ }^{2}$, and Kiwon Chong ${ }^{2}$ \\ ${ }^{1}$ Ubiquitous Computing Middleware Team, ETRI, Daejeon, Korea \\ kylee@etri.re.kr \\ ${ }^{2}$ Department of Computing, Soongsil University, Seoul, Korea \\ \{bluewj, sespop\}@empal.com, chong@ssu.ac.kr
}

\begin{abstract}
A technique for automatic code generation of USN applications based on Nano-Qplus is proposed in this paper. Nano-Qplus is a sensor network platform developed by ETRI. Programs of nodes such as sensors, routers, sinks and actuators in a sensor network are automatically generated through the technique. Developers can implement USN applications from models of sensor networks. The execution code is generated by setting attribute values of each node according to the model through the script proposed in this paper. Through the technique of this paper, developers can easily implement USN applications even if they do not know the details of low-level information. The development effort of USN applications also will be decreased because execution codes are automatically generated. Furthermore, developers can perform early test through rapid code generation, so the verified code is generated by correcting errors in the early development stage.
\end{abstract}

\section{Introduction}

Ubiquitous sensor network (USN) is a wireless network which consists of a lot of lightweight, low-powered sensors. A lot of sensors which are connected to a network sense geographical and environmental changes of the field. They transmit the sensing data to a base station and the data is transmitted to users through sensor network server. Collection of information in USN is performed through this process. Through USN, things can recognize other things and sense environmental changes, so users can get the information from the things and use the information anytime, anywhere. The sensor networks can be used for various application areas such as home, health, and robot.

However, it is difficult to construct USN applications. Resources of nodes in a sensor network are limited and wireless communication between nodes is unreliable. Nodes should also perform low-power operations. Developers should consider these facts, so it is very difficult to construct USN applications. Therefore, it is need to make developers can simply design USN applications by abstracting the details of low-level communication, data sharing, and collective operations.

Accordingly, a technique for automatic code generation from a simple design of USN application is proposed in this paper. Programs of nodes such as sensors,

\footnotetext{
* This work was supported by the Soongsil University Research Fund.
} 
routers, sinks and actuators in a sensor network are automatically generated by setting attribute values of a script proposed in this paper. Therefore, developers can easily develop USN applications even if they do not know the details of low-level communication, data sharing, and collective operations. The technique of this paper brings focus to USN application on a sensor network platform known as Nano-Qplus [1, 2].

\section{A Technique for Code Generation of USN Applications}

A programming model to construct USN applications based on Nano-Qplus is presented in this section. It is compared to the existing programming models for USN applications. Moreover, the script for the design of an application and the algorithm for automatic code generation of the application are presented.

\subsection{Concepts of the USN Programming}

Figure 1 presents the concept of USN programming described in existing works $[3,4$, $5,6,7,8]$. A modeling is done and a simple program based on the model is written using the high level language or the simple script. Then the code is automatically generated according to the program. It is important that the program is written using the high level language or the script. The high level language or the script helps users to construct applications, even though they do not know the details of low-level information of USN. A specification-level language, a script language, or APIs were proposed in order to abstract the low-level information in the related works. However, users should learn the proposed language, the script language or APIs in order to develop USN applications using these techniques.

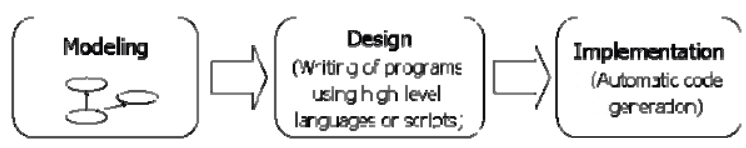

Fig. 1. The concept of USN programming in the existing works

A technique to complement the existing techniques for construction of USN applications is proposed in this paper.

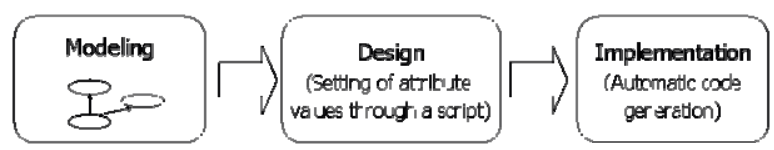

Fig. 2. The concept of USN programming in this paper

Figure 2 presents the concept of USN programming proposed in this paper. Developers can implement USN applications by automatically generating execution code of each node in the sensor networks after they do modeling the sensor networks and set 
attribute values of each node according to the model. The execution code is automatically generated by setting attribute values of each node through a script. Therefore, users can construct USN applications without learning a language or APIs.

\subsection{The USN Programming Model}

Figure 3 shows the USN programming model proposed in this paper. USN model is designed, and attribute values for sensor nodes, router nodes, sink nodes, and actuator nodes in the model are set through scripts. Modules and code templates which are provided by Nano-Qplus are selected according to the attribute values of scripts, so C codes for each node are automatically generated.

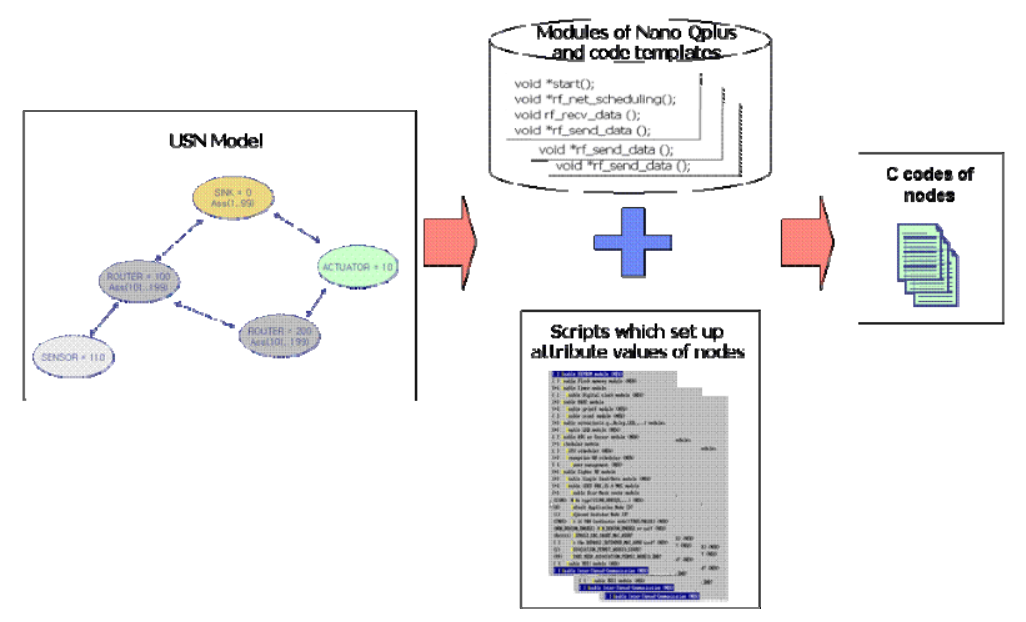

Fig. 3. USN programming model

Nodes in a USN communicate with each other through Store\&Forward method. Templates to generate programs of nodes according to that method are presented in this paper. Figure 4, 5, 6 and 7 show templates which contain modules provided by Nano-Qplus in order to generate programs for sensor nodes, router nodes, sink nodes, and actuator nodes.

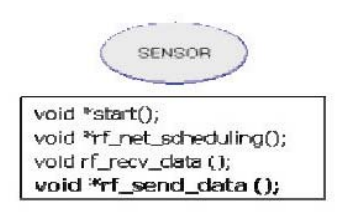

Fig. 4. Modules of a sensor node

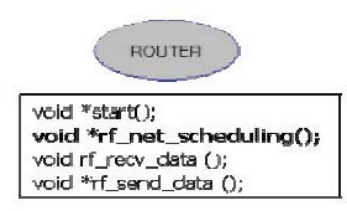

Fig. 5. Modules of a router node

Four types of nodes contain the same modules generally, but the contents of the modules are dependent upon the node type. For example, main role of a sensor node 
is to send sensing data to other nodes, so the contents of rf_send_data() module are dependent upon the script setting for the sensor node. Main role of a router node is to send data received from a node to other nodes, so the contents of rf_net_scheduling() module are dependent upon the script setting for the router node. Moreover, main role of a sink node and an actuator node is to process data, so the contents of rf_recv_data() module are depedent upon the script setting for the sink node and the actuator node.

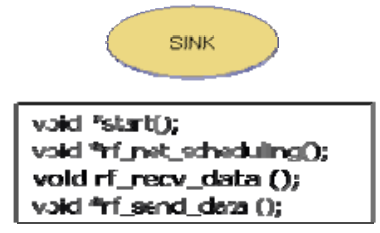

Fig. 6. Modules of a sink node

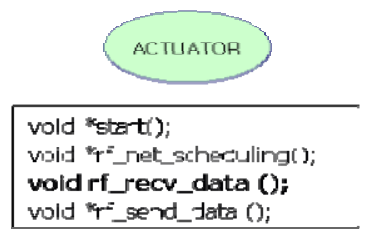

Fig. 7. Modules of a actuator node

\subsection{A Script for Generation of USN Applications}

To generate the application from the designed model, the script which can easily configure the attributes of each node in USN is proposed. Figure 8 shows the script.

The script has been written as a typical script language [9] which is used for configuring environment in Linux, so most of users can easily use it. If users configure the attributes of each node using the script, a program code for each node is automatically generated based on the script.

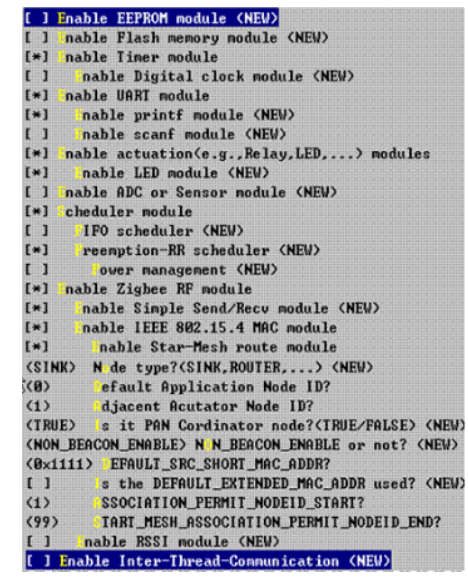

Fig. 8. A script for automatic code generation

The script showed in figure 8 has been written for generating the application on a sensor network platform known as Nano-Qplus. 


\subsection{An Algorithm for Generation of USN Applications}

The following is the process for generating source code to control each node based on the script.

Step 1 - Read Config_Info(.config) file in order to get the attribute values of a node.

Step 2 - Parse Config_info(.config) file and find out selected modules. Then read headers, data and function codes from HashTable_Module according to the selected modules and save them to the template.

Step 3 - Read main code from HashTable_Main based on selected modules and save it to the template.

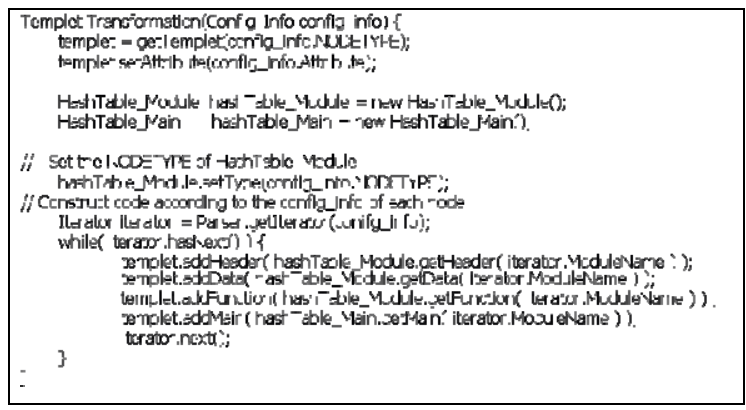

Fig. 9. An algorithm for generating USN application

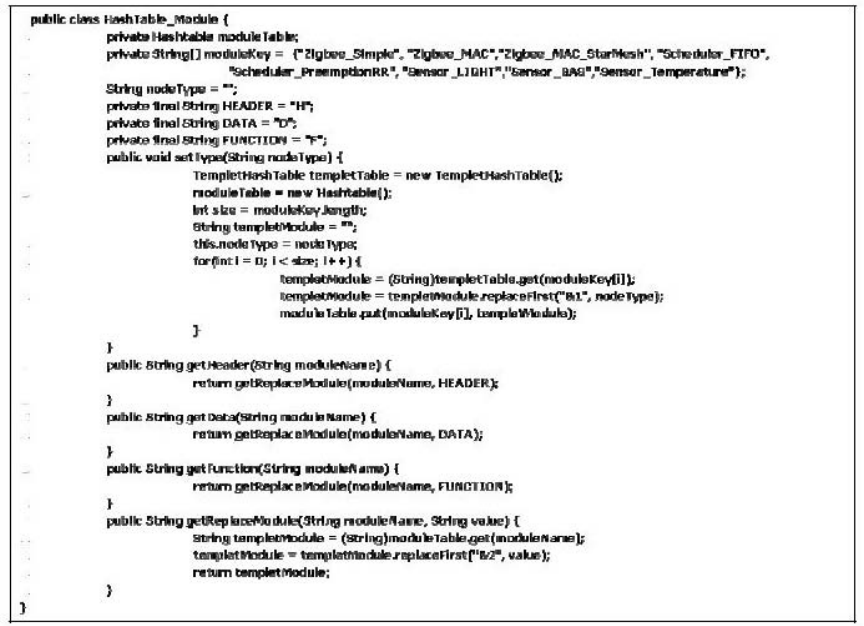

Fig. 10. HashTable_Module class

Figure 9 presents the algorithm for generating source code of each node. Headers, data and function codes are generated by calling the functions of HastTable_Module class according to the type of the target node. 
The HashTable_Module class used in the algorithm is presented in figure 10. The class includes getHeader(), getData() and getFunction() to generate the program for each node. These functions use the key and the value corresponding to the key defined in a hash table through the getReplaceModule() function in order to get source codes. HashTable_Module class generates the proper source code using a hash table dynamically because the codes of headers, data and functions are dependent upon the type of the node.

Table 1. Structure of hash table

\begin{tabular}{|l|l|}
\hline \multicolumn{1}{|c|}{ Key } & \multicolumn{1}{c|}{ Value } \\
\hline Zigbee_Simple & "\&1_Zig_Simple_\&2" \\
\hline Zigbee_MAC & "\&1_Zig_MAC_\&2" \\
\hline Zigbee_MAC_StarMesh & "\&1_Zig_StarMesh_\&2" \\
\hline Scheduler_FIFO & "\&1_Sche_FIFO_\&2" \\
\hline Scheduler_PreemptionRR & "\&1_Sche_PreemptionRR_\&2" \\
\hline Sensor_LIGHT & "\&1_Sensor_LIGHT_\&2" \\
\hline Sensor_GAS & "\&1_Sensor_GAS_\&2" \\
\hline Sensor_Temperature & "\&1_Sensor_Temperature_\&2" \\
\hline
\end{tabular}

It is necessary to define many hash tables according to the number of node type if a static hash table which has the keys and values for one node type is used. Moreover, the codes to control the hash tables must be written additionally according to the number of hash tables. It is necessary to define a new hash table and to write the code to control the hash table if a new type of the node is added.

However, there is no need to define a new hash table when a new type of the node is added if a dynamic hash table is used. No additional codes are needed such as control codes for hash tables. The structure of dynamic hash table used in the HashTable_Module class is presented in table 1. The key of the hash table is the name of a module provided by Nano-Qplus. Strings such as "\&1" and "\&2" in the key value are dynamically replaced according to the type of module and node. When the type of each node is determined, the string " $\& 1$ " is replaced with the type, and "\&2" is replaced with " $\mathrm{H}$ " (means Header), "D" (means Data) or "F" (means Function) based on the type of required module. Following is an example.

i.e.) If the Zigbee_Simple module for radio frequency communication of a node is selected, "\&1_Zig_Simple_\&2" value is selected from the hash table. Then, the value is replaced as follows by calling functions of HashTable_Module class.

setType("SINK"); $\rightarrow$ "SINK_Zig_Simple_\&2"

getHeader("Zig_Simple") $\rightarrow$ "SINK_Zig_Simple_H"

getFunction("Zig_Simple") $\rightarrow$ "SINK_Zig_Simple_F"

"SINK_Zig_Simple_H" is the name of a file which contains header codes of the ZigBee_Simple module for a sink node, and "Sink_Zig_Simple_F" is the name of a file which contains function codes of the ZigBee_Simple module for a sink node. 


\section{Case Study with Gas Monitoring System}

An USN application for Gas Monitoring System such as figure 11 has been developed using the proposed technique in this paper.

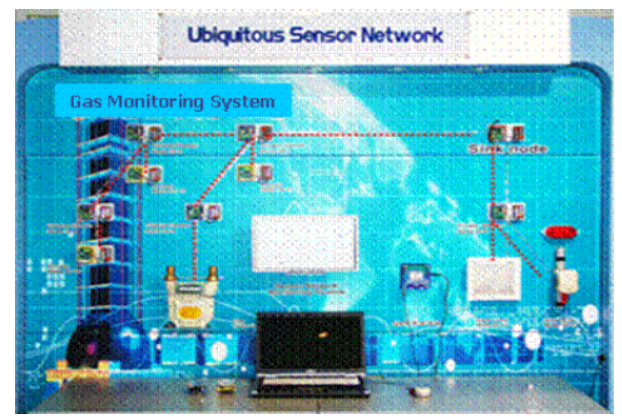

Fig. 11. Gas Monitoring System

A USN model was designed for the Gas Monitoring System. In the model, sensor nodes sense gas data and transmit the data to router nodes. The router nodes receive the data and transmit it to the sink node. The sink node receives the data, computes it and transmits it to the actuator node. The actuator node performs an action according to the threshold value.

The system of figure 11 was developed after the application was automatically generated using a script based on designed model. Result that applies, sensing data was forwarded from sensor node to router node and router node sent forwarded data to sink node. An action command according to a gas value was forwarded from sink node to router node and router node sent forwarded the action command to actuator.

The figure 12 is an example to set the attribute values of the script in order to generate automatically the program of the sink node. The USN application was automatically generated by setting the attribute values of each node through the script.

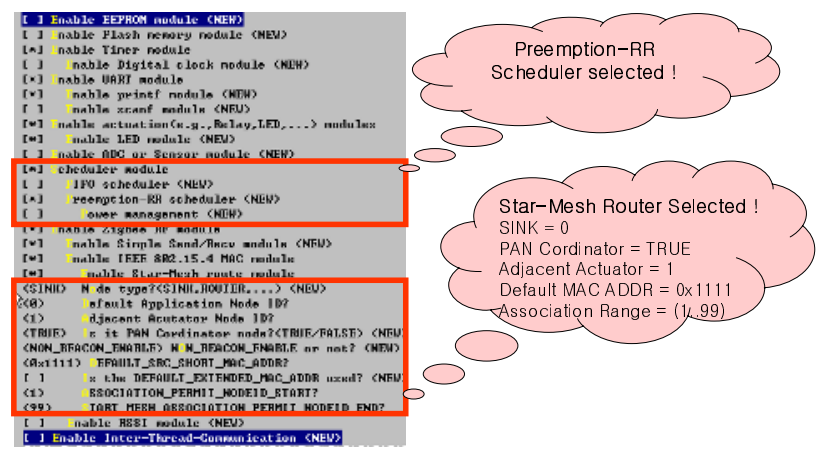

Fig. 12. An example of attribute values setting using the script 


\section{Conclusion}

The technique for automatic code generation of USN applications based on NanoQplus is proposed in this paper. Developers can implement USN applications by automatic generation of execution code of each node in the sensor networks after they make models of the sensor networks and set attribute values of each node according to the model using the script. The script for automatic code generation of each node is proposed in this paper. The templates and an algorithm for automatic code generation are also presented. Through the technique of this paper, developers will easily implement USN applications even if they do not know the details of low-level communication, data sharing, and collective operations. The development effort of USN applications also will be decreased because execution codes are automatically generated. Furthermore, developers can perform early test through rapid code generation, so the verified code is generated by correcting errors in the early development stage.

\section{References}

1. Kwangyong Lee et al., "A Design of Sensor Network System based on Scalable \& Reconfigurable Nano-OS Platform," IT-SoC2004, October 2004.

2. ETRI Embedded S/W Research Division, "Nano-Qplus," http://qplus.or.kr/

3. E. Cheong, J. Liebman, J. Liu, and F. Zhao, "Tinygals: a programming model for eventdriven embedded systems," SAC, 2003.

4. M. Welsh and G. Mainland, "Programming sensor networks using abstract regions," NSDI, 2004.

5. R. Newton and M. Welsh, "Region streams: Functional macroprogramming for sensor networks," DMSN, 2004.

6. A. Boulis, C. Han, and M. B. Srivastava, "Design and implementation of a framework for efficient and programmable sensor networks," MobiSys, 2003.

7. B. Greenstein, E. Kohler, and D. Estrin, "A sensor network application construction kit (SNACK)," SenSys, 2004.

8. Ramakrishna Gummadi, Omprakash Gnawali, and Ramesh Govindan, "Macroprogramming Wireless Sensor Networks Using Kairos," LNCS 3560, pp. 126-140, 2005.

9. Neil Matthew, Richard Stones, "Beginning Linux Programming 3rd Edition," WROX PRESS, 2003. 